This is an Accepted Manuscript of an article published by Taylor \& Francis in Journal of Interprofessional Care on 06 Feb 2016, available online: http:// www.tandfonline.com/13561820.2015.1072803

\title{
A sociological exploration of the tensions related to interprofessional collaboration in acute care discharge planning
}

\author{
Joanne Goldman \\ University of Toronto, Canada \\ Scott Reeves \\ Kingston University \& St George's, University of London, UK \\ Robert $\mathrm{Wu}$ \\ University of Toronto, Canada \\ Ivan Silver \\ University of Toronto, Canada \\ Kathleen MacMillan \\ University of Prince Edward Island, Canada \\ Simon Kitto \\ Ottawa University, Canada
}

\begin{abstract}
Patient discharge is a key concern in hospitals, particularly in acute care given the multifaceted and challenging nature of patients' health care needs. Policies on discharge have identified the importance of interprofessional collaboration, yet research has described its limitations in this clinical context. This study aimed to extend our understanding of interprofessional interactions related to discharge in a general internal medicine setting by using sociological theories to illuminate the existence of, and interplay between, structural factors and micro-level practices. An ethnographic approach was employed to obtain an in-depth insight into health care providers' perspectives, behaviours, and interactions regarding discharge. Data collection involved observations, interviews and document analysis. Approximately 65 hours of observations were undertaken, 23 interviews were conducted with health care providers, and government and hospital discharge documents were collected. Data were analyzed using a directed content approach. The findings indicate the existence of a medically dominated division
\end{abstract}


of healthcare labour in patient discharge with opportunities for some interprofessional negotiations; the role of organizational routines in facilitating and challenging interprofessional negotiations in patient discharge; and tensions in organizational priorities that impact an interprofessional approach to discharge. The findings provide insight into the various levels at which interventions can be targeted to improve interprofessional collaboration in discharge while recognizing the organizational tensions that challenge an interprofessional approach.

Key words: interprofessional collaboration, ethnography, acute care, patient discharge, social theory, medical dominance, negotiation

\section{Introduction}

Discharge planning is a complex event and a major priority in hospitals. Administrators and health care providers are particularly focused on minimizing patient length of stay, ensuring a safe and high quality patient discharge experience and reducing unnecessary readmissions (Connolly et al., 2009; Glasby, Littlechild, \& Pryce, 2006; Ontario Ministry of Health Care and Long Term Care, 2010). The concern with length of stay is linked to organizational imperatives concerning patient flow, and the aims to optimize bed availability, minimize emergency department wait times and reduce costs (Office of the Auditor General of Ontario, 2010; Wong et al., 2009). The emphasis on patient safety and quality of care reflects the potential for adverse events to occur during this period of transition and the goal to minimize readmissions (Davis, Devoe, Kansagara, Nicolaidis, \& Englander, 2012; Romagnoli, Handler, Ligons, \& Hochheiser, 2013). The planning 
and implementation of patient discharge is therefore a complex process involving multiple healthcare workers, driven by a range of, and possibly competing, health, political and economic factors. Discharge planning in general internal medicine (GIM) units is particularly complex given the multifaceted and challenging nature of patients' health and social care needs, and the varied discharge destinations of this patient group (Szecket, Wong, Wu, Berman, \& Morra, 2012). Policies on hospital discharge have identified the importance of interprofessional collaboration, however, recent research has described limitations with teamwork and collaboration in this clinical context (Greysen, Schiliro, Curry, Bradley, \& Horwitz, 2012a; Toronto Central LHIN Discharge Planning Steering Group, 2011).

Research on interprofessional interactions and discharge in GIM and other acute care hospital contexts has begun to illuminate challenges around effective teamwork, collaboration and communication. For example, in an interview study undertaken by Greysen, Schiliro, Horwitz, Curry \& Bradley (2012b) with physicians in GIM, participants reported diverse discharge 'team' experiences; some were described as fragmented, lacking cooperation and characterized by last-minute problem-solving while other discharge experiences were described as cohesive. Although physicians described teamwork as important to discharge, they did not necessarily know how to lead or function effectively within a team, which in turn was described as contributing to experienced conflict and inefficiency. The reporting of different 'team' experiences is a key theme in other studies that included a range of healthcare professional participants working in acute healthcare contexts. Pethybridge (2004) reported on unidisciplinary, 
multidisciplinary and transdisciplinary types of leadership and teamworking, each type suggested variations in how healthcare professionals interacted and worked together. A repeated theme in these studies on discharge in acute care is a lack of clarity about health care professionals' roles and responsibilities and problems with communication between professionals, with a shared concern that no one takes overall responsibility for discharge (Connolly et al., 2009; Greysen et al., 2012b; Pethybridge, 2004; Wong et al., 2011). Problems with communication and discharge have been attributed to professional hierarchy and insufficient contact among professionals (Greysen et al., 2012b; Wong et al., 2011). Factors identified as important to effective interprofessional collaboration and teamwork in discharge include sharing of information, trust, respect, communication, a learning culture and facilitative leadership (Pethybridge, 2004; Reeves, Lewin, Espin \& Zwarenstein 2010).

\section{Background}

Sociological theories of medical dominance (Freidson, 1976; 1988; Willis, 1989) and negotiated order (Strauss, Schatzman, Ehrlich, Bucher, \& Sabshin, 1963) have been applied to provide insight into the nature of macro level structural factors and micro level interprofessional interactions in different health care contexts. The focal point of theories of medical dominance is that physicians occupy a dominant position in the health care division of labour that affords them autonomy over their own work and the work of other clinical groups (Freidson, 1988; Willis, 1989). Over the past few decades there has been debate about the continued degree of medical dominance given political, social and technological changes (Boyce, 2006; Broom, 2006; Nugus, Greenfield, Travaglia, 
Westbrook, \& Braithwaite, 2010), as well as the impact of dynamic professional boundaries due to changes in the healthcare workforce and patient safety concerns (Iedema et al., 2006; Nancarrow \& Borthwick, 2005). Structural and organizational changes provide the possibility for greater independence of health occupations such as physiotherapy, occupational therapy and social work that in turn re-draws professional boundaries (Boyce, 2006). Despite these broader shifts, medical dominance has been reported as still shaping the context of, and interactions in, discharge meetings. One significant effect is the suppression of the voices of certain health care providers in the presence of physicians (Connolly et al., 2010) and where physicians have the authority to discharge and social workers have the responsibility for planning discharge (Dill, 1995). However, research from other health care contexts shows that other health care providers can 'resist' medical dominance (Lewis \& Tully, 2009; Liu, Manias \& Gerdtz, 2013).

Medical dominance theories provide a structural perspective of the organization of the division of labour, but do not explain how individuals act and interact within these structures. Developed by Strauss and colleagues, the negotiated order perspective is a sociological theory which provides a complementary analytical approach to understanding the processes of interaction amongst health professionals by focussing on how social order in healthcare contexts is negotiated between individuals on an on-going basis (Strauss et al., 1963; Strauss, 1978). As such, negotiation involves "the continual working out, together of who [is] to do what, how and with whom" (Strauss, 1978, p. 107). Negotiations may be co-operative or conflictual, and are more likely to occur when rules and policies are not definitive. However, not everything is equally negotiable or 
negotiable at all, as certain structural conditions, including institutional policies and regulations, can limit the actions and aims of the negotiating parties (Allen, 1997; Martin, Currie, \& Finn, 2009; Strauss, 1978; Svensson, 1996). The negotiated order approach can provide insights to the multiple dimensions and nuances of power, viewed as being diverse and distributed rather than uni-directional and static (Nugus et al., 2010). Recent research has shown instances of how negotiation may or may not occur amongst a range of health care providers (Allen, 1997; Miller \& Kontos, 2013; Reeves et al., 2009) and that patient management is often a key site of negotiation about clinical roles (Nugus et al., 2010).

This paper reports on a study that aimed to elicit a more in-depth understanding of interprofessional interactions in discharge in GIM by using two sociological perspectives to help understand the interplay of the structural factors and micro-level practices.

\section{Methods}

An ethnographic approach was employed to obtain in-depth insight into health care providers' perspectives, behaviours, and interactions regarding discharge in a GIM unit (Hammersley \& Atkinson, 2007; Reeves, Peller, Goldman, \& Kitto, 2013). This methodology was suitable given its focus on both activities and individuals' accounts of those activities as well as the exploratory nature of the study (Hammersley \& Atkinson, 2007). The first author (JG) undertook observations, conducted interviews, and examined relevant documents over a period of 18 months from January 2012 through to May 2013 in an observer role (Green \& Thorogood, 2004). As a non-clinician, JG did not 
participate in any clinical activities but did engage, when feasible, in informal discussions to raise questions or discuss observations.

\section{Setting}

This study was undertaken in the GIM unit of an academic teaching hospital in Canada. The unit provided care for patients with complicated medical problems and follow-up for patients discharged from emergency and medical and surgical wards. At the time of data collection, the ward admitted approximately 350 patients per month and had approximately 50 to 70 nurses, 16 medical residents, 12 attending physicians, four social workers, pharmacists and community care access centre case managers, three physiotherapists and occupational therapists, and one speech language pathologist, dietitian and spiritual care worker. The nursing group included two nurse managers, patient care coordinators and clinical nurse specialists, and the bedside nurses. A group of bedside nurses took turns being in-charge nurses.

The ward was a designated clinical teaching unit. The unit was composed of four medical teams, each consisting of one attending physician (the physician responsible for the medical team) and senior resident, two or more junior residents and two to four students. There was a fifth team of hospitalists. The attending physicians rotated every two to four weeks, consistently attending with the same team (e.g. Team \#5). Each GIM team had a social worker, physiotherapist, occupational therapist and pharmacist aligned with it. The nurses and other health care providers cared for patients on multiple teams. The hospital patient flow and utilization of care coordinators worked with all hospital units. Structured 
interprofessional care rounds organized to improve discharge planning occurred weekday mornings. The in-charge nurses attended rounds alongside the patient care coordinators and nurse managers. This group of nurses represented the bedside nurses at rounds.

\section{Data Collection}

The observations were undertaken covering both formal and informal activities, including daily interprofessional, medical and nursing rounds, monthly interprofessional and patient safety rounds, interactions at nursing stations, and shadowing of clinicians. Observations focused on verbal and non-verbal interprofessional interactions about discharge planning as well as general collaborative practice issues. Observations of intraprofessional interactions were also collected when pertinent to discharge. In total, approximately 65 hours of fieldwork data were gathered, covering different times during the weekday from 7:00 am to 4:30 pm. The observations were largely undertaken during the daytime when the range of healthcare providers was most likely working in the unit and there was activity concerning discharge. Handwritten fieldnotes were made during the observations; as soon as possible afterwards the notes were typed up with greater detail including asides, commentaries and analytic memos (Emerson, Fretz, \& Shaw, 2011; Hammersley \& Atkinson, 2007).

One-to-one semi-structured interviews were conducted following an initial three-month period of observations and then continued during the study period. The interviews involved questions about individuals' perceptions of their own and others' roles concerning discharge, the discharge process, interprofessional communication during 
discharge, and organizational discharge policies. A maximum variation sampling approach (Patton, 2002) was used with the aim to interview individuals representing each professional group working in GIM. Interview participants were purposively selected based on their professional group from the staff directory and recommendations made by participants. Twenty-three interviews, including one interview with two participants and four follow-up interviews, were conducted. Participants consisted of five nurses, three attending (senior) physicians, two medical residents (junior physicians), two social workers, two patient flow and utilization of care coordinators, and one physiotherapist, occupational therapist, pharmacist, speech language pathologist, community care access centre case manager and spiritual care worker. Two healthcare providers invited to participate in an interview did not respond. The sampling and timing of interviews were based on emerging ideas about the findings and relationships amongst concepts (Gobo, 2007). All interviews were audio recorded and transcribed verbatim.

As part of data collection, publicly available (online) provincial government and hospital documents about discharge were accessed. Data collection ceased when it was determined that the range of professional perspectives were represented in the interview data, the different spaces in the GIM unit relevant to patient discharge discussions and interactions had been observed, and accessible hospital and provincial government documents about discharge had been obtained.

Analysis 
Data collection and analysis occurred iteratively. The research team met regularly during the data collection process to reflect on emerging themes and plan for future observations and interviews accordingly. The data were coded using a directed content approach (Hsieh \& Shannon, 2005). In this approach, analysis begins with theory or relevant research findings as guidance for initial codes, and in turn aims to further refine, extend, and enrich the theory. For this study, the theories of medical dominance (particularly the concepts of authority and autonomy) and negotiated order provided guidance for coding the data. Data, method and theory triangulation allowed for the development of a more comprehensive understanding of the phenomena of interprofessional interactions in discharge in GIM (Flick, 1992; Kitto, Chesters, \& Grbich, 2008). The collection of interview data from varied health care providers and observation data from a range of areas and events in the unit, in addition to the collection of a combination of observation, interview and documentary data, allowed the study to gain insight to the practice of discharge from different professional perspectives, how it was practiced in different places, and to compare how people talked about discharge in relation to their actual behaviours. Furthermore, the selection of the theories of medical dominance and negotiated order enabled analysis and interpretation of the data through different theoretical lenses.

\section{Ethical considerations}

Ethics approval for this study was received from the hospital research ethics board where the study took place. Consent was obtained from each interview participant and JG explained the study during periods of observation whenever feasible. 


\section{Findings}

The first part of the findings reports on the existence of a medically dominated approach

to patient discharge, as well as the opportunities for interprofessional negotiations within this broader structure. The second section explores the role of organizational routines in facilitating and challenging interprofessional negotiations in patient discharge. The third section reports on competing organizational priorities and the implications for an interprofessional approach to discharge.

\section{Division of healthcare labour and discharge}

Hospital discharge policy outlined physicians' authoritative role in discharge. According to policy, physicians were responsible for discharge but it was also noted that decisionmaking about discharge should occur in collaboration with the interprofessional care team:

Discharge readiness is determined by the assessment and decision that the patient no longer requires the intensity of resources/services in acute care... This decision is made by the most responsible physician (MRP), in collaboration with the interprofessional care team.

The attending physician or designate writes a discharge order and communicates the order to the patient and relevant staff. (Hospital policy, p. 1, p. 2)

Participants' perceptions and behaviours reflected these policies, indicating that there was a shared understanding amongst all healthcare providers that physicians had control over discharge decision making and that social workers, physiotherapists, occupational therapists and pharmacists, amongst other healthcare workers, played a 'supportive' role to the physicians' overall management of this process: 
I think the role of most of those people (nurses, therapists, etc.) is to assess and to decide what happens and then give a recommendation. And then it's up to the physician to piece it all together and decide what to do with it. (Physician, Interview \#22)

It's not my role to say a patient can go home. I can say physically whenever they're medically stable then they're functionally fit to go...I can't discharge a patient from hospital. So the lead comes from them. (medical team). (Physiotherapist, Interview \#9)

Accordingly, the discharge of a patient in this GIM unit was conceptualized as a patient's 'medical', 'social' and 'functional' readiness for discharge; the first was identified as being within the physicians' domain while the latter two were recognized as the professional responsibilities of social workers, and physiotherapists and occupational therapists. The social workers reported that discharge planning always took precedence over other roles such as counselling, and that discharge related activities had increased over the years, which consumed the majority of their work time. One experienced social worker attributed this emphasis on discharge to the increasing volume of patients admitted with complex health and social problems. The increasing involvement of social workers in discharge, though, provided them with the opportunity to be more assertive in their input to physician decision-making about discharge as shown in the following example:

The social worker is explaining to the physicians that cannot do a capacity evaluation of a patient until off antibiotics. The doctor says "Will be on antibiotics for 3 weeks, so she'll be in the hospital until then?" The social worker says "Yes, evaluation won't hold up in courts if on antibiotics." (Fieldnotes, May 7, 2012, morning rounds)

The physiotherapists and occupational therapists' professional roles involved the assessment of a patient being physically and cognitively ready to be discharged from the hospital. The following data provides one of numerous examples of medical attendings 
and residents seeking out the input of these professionals during rounds to establish whether they thought the patient was 'safe' to be discharged:

Doctor: PT and OT were happy with his progress, almost ready for discharge. Physiotherapist: We want to see him on stairs again.

Doctor: Hope for later today once PT gives green light.

Physiotherapist: Hopefully. (Fieldnotes, May 7, 2012, morning rounds)

The pharmacists described their role in discharge in relation to medication reconciliation and explained that due to a heavy workload, they could not be involved in the discharge of every patient. They described prioritizing patients with particular needs such as elderly people who had trouble managing, or had changes made to, their medications. Their expertise overlapped with the physicians; either profession could complete the discharge reconciliation but the physicians were required to authorize it.

The social workers, physiotherapists, occupational therapists and pharmacists were not aware of the particular hospital policies that affected their professional-specific discharge activities, but observations of their behaviours reflected the impact of organising discourses around discharge, patient flow, and patient safety, which likely created opportunities for them to be more influential in discharge. For example, the occupational therapists and physiotherapists reported communicating to the medical team their assessment of a patient as demonstrated in the following quote. This example indicates these professionals' understanding of the competing organizational priorities of a timely and safe discharge and the opportunity to inform physicians' decision making by attending to these imperatives:

If we see someone and we 're really busy we try to send either a quick email or a clinical message to the team so that they have the heads up from a functional perspective they might not be at their baseline, they need a little bit more time so 
that they're not going in blind expecting to discharge them.

(Occupational therapist, Interview \#13)

In contrast, the nurse managers did not view themselves as being involved in day-to-day discharge activities, but worked at a 'higher level' to ensure that the GIM unit met organizational metrics and goals concerning patient safety and discharge. However, they did express a commitment to represent bedside nurses when needed, such as during morning rounds. The data demonstrated more ambiguity in relation to the bedside nurses' roles in relation to discharge. The bedside nurses were encouraged by the nurse managers and patient care coordinators to speak directly to the physicians about discharge issues, but as a clinical nurse specialist explained, their comfort in doing so depended on the particular context. The bedside nurses worked across medical teams and therefore they did not have the same relationships with a 'team' as the occupational therapists, physiotherapists, social workers and pharmacists. As the following quote indicates, the bedside nurses' expertise in patient discharge was not as clearly articulated as the other healthcare providers, reflecting a more traditional hierarchical division of healthcare labour:

...if an INR (laboratory measurement of how long it takes blood to form a clot) was really low, a nurse would call a [medical] team and say no, the patient can't be discharged because that's something really concrete. But if the nurse just felt that the patient, you know, the patient had said to her I can't cope at home, the nurse may not report that. (Nurse, Interview \#8)

The social workers, physiotherapists and occupational therapists agreed that the medical teams would usually not discharge a patient if one of them identified the patient as not safe for discharge. On the other hand, participants reported that the nurses did not have this same influence on discharge decision making. 


\section{Organizational routines and negotiations}

The division of healthcare labour in discharge required ongoing negotiations amongst the physicians, social workers, physiotherapists, occupational therapists, pharmacists and nurses as they worked within the medically dominated interprofessional approach to discharge. The unit's organizational routines of morning rounds, referrals, and informal interactions both supported and challenged the opportunities for, and effectiveness of, interprofessional interactions.

Rounds. The morning care rounds took place in a meeting room and were attended by the range of healthcare providers working on the unit and the hospital patient flow and utilization of care coordinators. The rounds had been designed to support efficient patient discharge and had a tightly managed structure where all patients on a medical team were reviewed within a 15-minute time period. While some physicians spoke in a positive manner about the 'efficiency' of rounds, other physicians as well as social workers, occupational therapists, and physiotherapists, expressed concerns about the limited opportunities for meaningful interprofessional interactions about discharge. The following observation shows an example of a limited interprofessional interaction about patient discharge during rounds and the resulting sideline conversations that occurred:

Physician says about a patient "It would be great if we can get her out later this week". Social worker, occupational therapist and physiotherapist make faces at each other seemingly indicating their disagreement with something physician is saying. Social worker says that has not started application yet and gives reason concerning patient details. Rounds continue but I notice there is quiet side talk amongst these three health care providers. (Fieldnotes January 28, 2013, morning rounds) 
The nurse managers, patient care coordinators, and in-charge nurses attended rounds and aimed to present and support nursing perspectives concerning discharge; for example, a patient care coordinator reported on nursing leaders' efforts to "back each other up...make them (junior nurses) feel comfortable" (Nurse, Interview \#2). However, the bedside nurses did not participate in these rounds. The in-charge nurses and patient care coordinators were responsible for obtaining input from bedside nurses prior to the rounds and then reporting back afterwards. The nurses described the challenges of this process of information sharing given the large number of bedside nurses and patients in the unit: ...we do our best to put our two cents in....Because we have 38 patients and there's myself or the in-charge are two people going into rounds we don't know exactly all the little details about the patients' perspectives. (Nurse, Interview \#16)

They therefore did not have the same opportunity to engage in discussions about discharge with the physicians. Problems with this lack of communication were apparent in observations of nursing rounds where there were instances where nurses had patient information that conflicted with the discharge decisions that had been made during rounds, as demonstrated in the following example:

The in-charge says patient going to nursing home today, possible discharge today. One of the nurses says they just started her on IV fluids. The charge nurse says 'what?' and then goes on to say this eating and feeding issue is going to be an issue and we're not going to solve it here. Some discussion ensues. There is seemingly miscommunication as charge nurse reporting that patient is being discharged but nurse reporting that has started IV fluids. (Fieldnotes, May 24, 2012, nursing rounds)

Referrals. The social workers, occupational therapists and physiotherapists required some type of referral to be involved in a patient's care, and interactions around discharge. The medical team played a large role in referrals since they admitted patients to GIM yet there was also an increasingly interprofessional approach to referrals in the unit. Despite an 
interprofessional approach, these referrals still required negotiations with the medical team due to their coordinating role in patient care and discharge. There was much discussion amongst social workers, occupational therapists and physiotherapists about 'appropriate' and 'timeliness' of referrals, which were seen to vary by medical attending and team. For example, one physician commented that attendings had different practices with interprofessional referrals due to perceptions that involving other health care providers can delay discharge. On the other hand, these other professionals discussed how missed, inappropriate or delayed referrals could negatively impact on patient discharge:

I think in general they're (medical team) pretty good at recognizing that we need to see them at some point but I think we could probably be referred earlier on than a lot of them would refer... just because somebody's on oxygen or whatever doesn't mean we can't get in there and do something with them or at least...get the ball rolling. (Occupational therapist, Interview \#13)

The pharmacists were involved in medication related issues for all patients on their team; they discussed needing to set boundaries with the medical team as sometimes residents wanted pharmacists to be involved in activities such as discharge reconciliation more than they could due to other patient responsibilities.

Routine interactions. Interactions during the day were largely informal or ad hoc opportunities. The pharmacists for example described listening in on the medical team's informal meeting in the hallway following rounds to negotiate their role in discharge reconciliations for that day:

...it totally depends on the [medical] team and how actively involved you are and how much they look at you to help but after rounds I'd be like ok so these are the discharges, I can do a discharge rec for these ones, ok you take care of that one... (Pharmacist, interview \#15) 
The nurses and social workers, in particular, expressed concern about the lack of opportunity for negotiations with the medical team about discharge plans over the course of the day, given the frequency with which discharge plans changed during this time. This was reported as frustrating for nurses and social workers that interacted with the patients and family members in preparation for discharge. For example, a decision to discharge a patient could be reversed due to medical concerns. In these situations, while the health care providers recognized the complexities of patients' medical needs, the lack of negotiation opportunities was challenging, as a social worker noted:

...Sometimes it will be ok, I arranged for someone to pick this person up between 11 and 11:30 then they've arranged the landlord to meet them at home with their keys and this person's going to bring them some clothes... and you've done like every last detail for this person's life and then you tell the resident they're like oh yea no we just drew blood so we need another day and it's like ok, thanks, good to know. So that will happen all the time. (Social worker, Interview \#5)

The social workers, physiotherapists and occupational therapists were often observed sitting together at the nursing station discussing patients. The negotiating influence of this group of professionals could also be attributed to their development of an informal alliance with each other, which enhanced their perceived effectiveness in engaging in negotiations with the medical team. One of the social workers referred to this particular small group of healthcare providers as a "well oiled machine" in relation to negotiating discharge with their medical colleagues.

\section{Competing organizational priorities and negotiations}

The physicians explained that their decision making about discharge was affected by other professional responsibilities and hospital policies that could introduce tensions into 
negotiations about discharge with other healthcare providers. For example, physicians acknowledged challenges with the timing of the discharge and a lack of negotiation, explaining that this responsibility is one amongst others that need to be accomplished during the day:

...the nurses really want to know a specific time but...things are very unpredictable for us and we get caught up in like a code or something happens and then you can't do it and then it's like oh well now you're late, now they're upset because you haven't discharged them yet so then I think the reaction to that from a physician perspective is just not to create any expectation of discharge until you know you actually have time to do it... (Senior resident, Interview \#14)

Limited physician-nurse interaction due to, for example, these competing professionspecific duties, in turn affected nurses' ability to communicate with the patients and families and plan for discharge, which was reported to be frustrating for the nurses:

So the frustration comes in because you are that middle person...you're answering to the doctors getting upset at you why are you calling and you're answering to the family why isn't my family member being discharged. Where are the papers, you know, I've wasted my time. It gets frustrating. (Nurse, Interview \#11)

In addition, the physicians also described pressures to address organizational expectations concerning patient safety and patient flow. For example, it was noted that physicians have to manage the various factors that affect discharge decision making, which may impact on an interprofessional approach to discharge:

I think there's patient safety in terms of the safety for patients who have to stay in hospital and then there's patient safety for patients who are being discharged out of hospital to some other facility or home and I do think that a lot of the times the medical team is thinking about patient safety amongst their other patients as a stimulus to try to discharge people who have any hope of leaving hospital somewhere else. (Physician, Interview \#23)

The social workers, physiotherapists, occupational therapists and pharmacists discussed the complex trajectories of patients in GIM and the need to be flexible given competing 
priorities. For example, they described strategies undertaken to minimize duplication of efforts in relation to discharge activities given the frequency with which decisions about discharge changed. In these instances they valued communication to optimize interprofessional interactions and patient care in the discharge process.

\section{Discussion}

As outlined above, medical dominance, for the most part, shaped discharge practices in this GIM unit. However political, economic and patient factors created opportunities for social workers, physiotherapists, occupational therapists and pharmacists to more clearly define their roles in the healthcare division of labour. Nurse managers were involved in unit and organizational goals, and encouraged patient care coordinators and bedside nurses' activities regarding discharge; however, there was less recognition of patient care coordinators' and bedside nurses' roles and contributions to the discharge process. Given the complexity of decision making in discharge and the number of healthcare workers involved in this process, interprofessional negotiations were central to these interactions. Opportunities for these negotiations were dependent on rounds routines, referral practices and opportunistic interactions. In addition, negotiations were affected by competing clinical and organizational priorities.

These findings support the need to explore medical dominance not as a binary issue linked to whether certain health occupations and professions are gaining or losing power; rather, it is more helpful to investigate its complexity, given such issues as a managerial approach to discharge and evolving interprofessional dynamics (Broom, 2006; Correia, 
2013; Tousijn, 2006). Hospital discharge policies require physicians as well as other healthcare providers to be cognizant of, and work towards, timely and safe patient discharge in order to meet hospital and government requirements. This imperative has changed social workers' roles in the unit to being more heavily focused on patient discharge, has emphasized physiotherapists and occupational therapists' contributions to assessing a 'safe' patient discharge, and has strengthened pharmacists' roles in supporting safety in medications and patient discharge. Nurses are playing a major role in bed management (Allen, 2014), and data from this study support this phenomenon for nurse managers. The work of bedside nurses in GIM, though, reflects pressures for them to undertake activities required to free up beds (Hau, 2004), which generates frustration with the lack of opportunities to participate in decision making about patient discharge. These findings reflect the ongoing struggle related to the nursing professions' wider role in care giving (Allen, 2004; 2014), and encourages further attention to integrating bedside nurses into interprofessional interactions concerning discharge in GIM.

While, as noted above, structural factors created opportunities for changes in the division of healthcare labour in discharge, negotiations were needed to maintain, revise or challenge these professional boundaries given that physicians continued to have control over the discharge process. The opportunity for and effectiveness of negotiations were contingent on interactions to occur between these healthcare providers and physicians, through rounds, referrals and ad hoc interactions. The negotiations between the physicians and social workers, occupational therapists, physiotherapists and pharmacists contrasts with earlier work in GIM that showed more limited negotiations between 
physicians and these providers (Reeves et al., 2009). Despite the strong emphasis on discharge in rounds, there were some concerns that the interactions were largely focused on patient flow rather than on meaningful interprofessional interactions. Rounds in hospitals potentially have a range of educational, clinical and managerial objectives (Birtwistle, Houghton, \& Rostill, 2000; Wachter \& Verghese, 2012; Walton \& Steinert, 2010); the structure of rounds is therefore susceptible to an increasing focus on efficiency and patient flow that can compete with the goal of an interprofessional approach to care. Furthermore, researchers have reported that an increased use of technology is having an impact on face-to-face interactions. For example, the implementation of an electronic medical record in a hospital setting has been shown to decrease face-to-face communication between physicians and nurses and worsen overall agreement about the plan of care (Taylor, Ledford, Palmer, \& Abel, 2014); in addition, the use of short text messages in GIM has been linked to fewer verbal conversations and face to face interactions (Wu et al., 2014). Reflexivity about organizational routines such as rounds, referral practices and face-to-face and electronic communication can facilitate formal and informal interprofessional negotiations that contribute to high quality patient discharge.

The nursing leadership in this study expressed a desire to further integrate bedside nurses into interprofessional interactions yet confronted organizational barriers to doing so, such as nurses not being able to leave patients for the duration of rounds. The nurses also worked across medical teams in contrast to the social workers, occupational therapists, physiotherapists and pharmacists who were aligned with the medical teams. The interactions amongst this group of healthcare professionals is indicative of a premise of 
negotiated order theory that negotiations are often about the ability to argue and create alliances (e.g. Svennson, 1996). These professionals regularly consulted with each other prior to interacting with the physicians. These alliances were also apparent in relationships developed between these healthcare professionals and medical attendings, as reported in a related publication (Goldman et al., 2015). The nurses' scheduling, their larger number and turnover, were likely barriers to enabling them to develop such alliances.

The interprofessional negotiations in discharge occurred within a larger organizational context of professional responsibilities, hospital policies and managerial discourses, which impeded an interprofessional approach at times. This suggests the need to move beyond the descriptive 'teamwork' evaluations to recognize the key professional, social and political issues that underpin both the barriers and facilitators to interprofessional practice (Reeves et al., 2010). The theoretical analysis offered in this paper builds upon the initial work on interprofessional interactions and discharge in acute care showing various types of leadership and interprofessional interactions (Connolly et al., 2009; Greysen et al., 2012b; Pethybridge, 2004). By illuminating the structural factors and processual nature of interprofessional interactions, it provides direction for interventions to support an interprofessional approach to discharge while recognizing the existing tensions.

The limitations of this study are that the data were gathered from one GIM unit in Canada. This limits the transferability of findings, as discharge policy details, the 
organization of health care professionals in 'teams', and professional and interprofessional practices concerning discharge can differ in other hospitals.

Furthermore, while the sampling strategy aimed to capture a range of professional perspectives, it is possible that further interviews would have contributed additional insights to the themes presented. This study examined moments of discharge in the GIM unit but did not undertake case studies of particular patients throughout their hospital stay to discharge that could provide additional understandings, and should therefore be considered as a methodological approach for future research. Nevertheless, this study is helpful in beginning to describe and unpack the social factors and processes involved in interprofessional interactions in discharge in this particular clinical context, and provides a framework for future studies in other GIM settings. Examining interprofessional interactions around discharge in particular clinical settings is helpful in providing insight into commonalities and differences across clinical contexts.

\section{Acknowledgements}

We would like to thank the GIM staff that participated in the interviews and observations in this research.

\section{Declaration of interest}

The authors report no conflicts of interest. The authors alone are responsible for the writing and content of this paper. This research was funded by the Canadian Institutes of Health Research through a Frederick Banting and Charles Best Canada Graduate Scholarship Doctoral Award. 


\section{References}

Allen, D. (1997). The nursing-medical boundary: a negotiated order? Sociology of Health \& Illness, 19(4), 498-520.

Allen, D. (2004). Re-reading nursing and re-writing practice: towards an empirically based reformulation of the nursing mandate. Nursing Inquiry, 11(4), 271-283.

Allen, D. (2014). Inside 'bed management': ethnographic insights from the vantage point of UK hospital nurses. Sociology of Health \& Illness, 37(3), 370-384.

Birtwistle, L., Houghton, J., \& Rostill, H. (2000). A review of a surgical ward round in a large paediatric hospital: Does it achieve its aims? Medical Education, 34(5), 398-403.

Boyce, R. (2006). Emerging from the shadow of medicine: allied health as a 'profession community' subculture. Health Sociology Review, 15(5), 520-534.

Broom, A. (2006). Reflections on the centrality of power in medical sociology: an empirical test and theoretical elaboration. Health Sociology Review, 15(5), 496505.

Connolly, M., Grimshaw, J., Dodd, M., Cawthorne, J., Hulme, T., Everitt, S., . . Deaton, C. (2009). Systems and people under pressure: the discharge process in an acute hospital. Journal of Clinical Nursing, 18(4), 549-558.

Connolly, M., Deaton, C., Dodd, M., Grimshaw, J., Hulme, T., Everitt, S., \& Tierney, S. (2010). Discharge preparation: Do healthcare professionals differ in their opinions? Journal of Interprofessional Care, 24(6), 633-643. 
Correia, T. (2013). The interplay between managerialism and medical professionalism in hospital organisations from the doctors' perspective: A comparison of two distinctive medical units. Health Sociology Review, 22(3), 255-267.

Davis, M.M., Devoe, M., Kansagara, D., Nicolaidis, C., \& Englander, H. (2012). "Did I do as best as the system would let me?" Healthcare professional views on hospital to home care transitions. Journal of General Internal Medicine, 27, 1649-1656.

Dill, A.E. (1995). The ethics of discharge planning for older adults: An ethnographic analysis. Social Science \& Medicine, 41(9), 1289-1299.

Emerson, R.M., Fretz, R.I., \& Shaw, L.L. (2011). Writing ethnographic fieldnotes. Chicago: University of Chicago Press.

Flick, U. (1992). Triangulation revisited: Strategy of validation or alternative? Journal of Theory of Social Behaviour, 22(2), 175-198.

Freidson, E. (1976). The division of labor as social interaction. Social Problems, 23(3), 304-313.

Freidson, E. (1988). Profession of medicine: A study of the sociology of applied knowledge. Chicago: The University of Chicago Press.

Glasby, J., Littlechild, R., \& Pryce, K. (2006). All dressed up but nowhere to go? Delayed hospital discharges and older people. Journal of Health Services Research and Policy, 11(1), 52-58. 
Gobo, G. (2007). Sampling, representativeness and generalizability. In C. Seale, G. Gobo, J. F. Gubrium \& D. Silverman (Eds.), Qualitative research practice (pp. 405-426). London: Sage Publications Ltd.

Goldman, J., Reeves, S., Wu, R., Silver, I., MacMillan, K., \& Kitto, S. (2015). Medical residents and interprofessional interactions in discharge: an ethnographic exploration of factors that affect negotiation. Journal of General Internal Medicine. [Epub ahead of print]

Green, J., \& Thorogood, N. (2004). Qualitative methods for health research. London: Sage Publications Ltd.

Greysen, S.R., Schiliro, D., Curry, L., Bradley, E.H., \& Horwitz, L.I. (2012a). "Learning by doing" -resident perspectives on developing competency in high-quality discharge care. Journal of General Internal Medicine, 27(9), 1188-1194.

Greysen, S.R., Schiliro, D., Horwitz, L.I., Curry, L., \& Bradley, E.H. (2012b). "Out of sight, out of mind": Housestaff perceptions of quality-limiting factors in discharge care at teaching hospitals. Journal of Hospital Medicine, 7(5), 376-381.

Hammersley, M., \& Atkinson, P. (2007). Ethnography: Principles in practice (3rd edition). London: Routledge.

Hau, W.W. (2004). Caring holistically within new managerialism. Nursing Inquiry, 11(1), 2-13.

Hsieh, H.F., \& Shannon, S.E. (2005). Three approaches to qualitative content analysis. Qualitative Health Research, 15, 1277-1288. 
Iedema, R.A.M., Jorm, C., Long, D., Braithwaite, J., Travaglia, J., \& Westbrook, M. (2006). Turning the medical gaze in upon itself: Root cause analysis and the investigation of clinical error. Social Science \& Medicine, 62(7), 1605-1615.

Kitto, S.C., Chesters, J., \& Grbich, C. (2008). Quality in qualitative research. Medical Journal of Australia, 188(4), 243-246.

Lewis, P.J., \& Tully, M.P. (2009). Uncomfortable prescribing decisions in hospitals: the impact of teamwork. Journal of the Royal Society of Medicine, 102, 481-488.

Liu, W., Manias, E., \& Gerdtz, M. (2013). Medication communication during ward rounds on medical wards: Power relations and spatial practices. Health (London), $17(2), 113-134$.

Martin, G.P., Currie, G., \& Finn, R. (2009). Reconfiguring or reproducing intraprofessional boundaries? Specialist expertise, generalist knowledge and the 'modernization' of the medical workforce. Social Science \& Medicine, 68(7), 1191-1198.

Miller, K.L., \& Kontos, P.C. (2013). The intraprofessional and interprofessional relations of neurorehabilitation nurses: a negotiated order perspective. Journal of Advanced Nursing, 69(8), 1797-1807.

Nancarrow, S., \& Borthwick, A. (2005). Dynamic professional boundaries in the healthcare workforce. Sociology of Health \& Illness, 27(7), 897-919.

Nugus, P., Greenfield, D., Travaglia, J., Westbrook, J., \& Braithwaite, J. (2010). How and where clinicians exercise power: Interprofessional relations in health care. Social Science \& Medicine, 71, 898-909. 
Office of the Auditor General of Ontario. (2010). 2010 Annual report. Retrieved from http://www.auditor.on.ca/en/reports_en/en10/2010ar_en.pdf

Ontario Ministry of Health Care and Long Term Care. (2010). Ontario wait times. Retrieved from http://www.health.gov.on.ca/en/pro/programs/waittimes/edrs/alc_definition.aspx

Patton, M.Q. (2002). Qualitative research and evaluation methods ( $3^{\text {rd }}$ edition). Thousand Oaks, California: Sage Publications, Inc.

Pethybridge, J. (2004). How team working influences discharge planning from hospital: A study of four multi-disciplinary teams in an acute hospital in England. Journal of Interprofessional Care, 18(1), 29-41.

Reeves, S., Lewin, S., Espin, S., \& Zwarenstein, M. (2010). Interprofessional teamwork for health and social care. United Kingdom: Wiley-Blackwell.

Reeves, S., Peller, J., Goldman, J., \& Kitto, S. (2013). Ethnography in qualitative educational research: AMEE Guide No. 80. Medical Teacher, 35(8), E1365E1379.

Reeves, S., Rice, K., Conn, L. G., Miller, K. L., Kenaszchuk, C., \& Zwarenstein, M. (2009). Interprofessional interaction, negotiation and non-negotiation on general internal medicine wards. Journal of Interprofessional Care, 23(6), 633-645.

Romagnoli, K.M., Handler, S.M., Ligons, F.M., \& Hochheiser, H. (2013). Home-care nurses' perceptions of unmet information needs and communication 
difficulties of older patients in the immediate post-hospital discharge period. BMJ Quality \& Safety, 22(4), 324-332.

Strauss, A. L. (1978). Negotiations: Varieties, contexts, processes and social order. London: Jossey-Bass.

Strauss, A., Schatzman, L., Ehrlich, D., Bucher, R., \& Sabshin, M. (1963). The hospital and its negotiated order. In E. Freidson (Ed.), The hospital in modern society. New York: Free Press.

Svensson, R. (1996). The interplay between doctors and nurses — a negotiated order perspective. Sociology of Health \& Illness, 18(3), 379-398.

Szecket, N., Wong, H. J., Wu, R. C., Berman, H. D., \& Morra, D. (2012). Implementation of a continuous admission model reduces the length of stay of patients on an internal medicine clinical teaching unit. Journal of Hospital Medicine, 7(1), 55-59.

Taylor, S.P., Ledford, R., Palmer, V., \& Abel, E. (2014). We need to talk: an observational study of the impact of electronic medical record implementation on hospital communication. BMJ Quality \& Safety, 23, 584-588.

Toronto Central LHIN Discharge Planning Steering Group. (2011). Improving hospital discharge planning \& patient transitions in the Toronto Central LHIN. Retrieved from http://www.torontocentrallhin.on.ca/uploadedFiles/Home_Page/Report_and_Publi cations/Discharge\%20Planning\%20-\%20LS\%20ALC\%20report.pdf

Tousijn, W. (2006) Beyond decline: consumerism, managerialism and the need for a new medical professionalism. Health Sociology Review, 15(5), 469. 
Wachter, R.M., \& Verghese, A.C. (2012). The attending physician on the wards: finding a new homeostasis. Journal of the American Medical Association, 308(10), 977-978.

Walton, J.M., \& Steinert, Y. (2010). Patterns of interaction during rounds: implications for work-based learning. Medical Education, 44(6), 550-558.

Willis, E. (1989). Medical dominance: the division of labour in Australian health care. Sydney: Allen \& Unwin.

Wong, H., Wu, R.C., Tomlinson, G., Caesar, M., Abrams, H., Carter, M.W., \& Morra, D. (2009). How much do operational processes affect hospital inpatient discharge rates? Journal of Public Health, 31(4), 546-553.

Wong, E.L., Yam, C.H., Cheung, A.W., Leung, M.C., Chan, F.W., Wong, F.Y., \& Yeoh, E.K. (2011). Barriers to effective discharge planning: a qualitative study investigating the perspectives of frontline healthcare professionals. BMC Health Services Research, 11, 242.

Wu, R., Appel, L., Morra, D., Lo, V., Kitto, S., \& Quan, S. (2014). Short message service or disService: Issues with text messaging in a complex medical environment. International Journal of Medical Informatics, 83(4), 278-284. 